\title{
Probiotics and the Microbiota-Gut-Brain Axis: Focus on Psychiatry
}

\author{
Sabrina Mörkl ${ }^{1,2}$ (D) Mary I Butler ${ }^{1,3} \cdot$ Anna Holl $^{1} \cdot$ John F Cryan ${ }^{1,4} \cdot$ Timothy G Dinan ${ }^{1,3}$
}

Published online: 13 May 2020

(C) The Author(s) 2020

\begin{abstract}
Purpose of Review Probiotics are living bacteria, which when ingested in adequate amounts, confer health benefits. Gut microbes are suggested to play a role in many psychiatric disorders and could be a potential therapeutic target. Between the gut and the brain, there is a bi-directional communication pathway called the microbiota-gut-brain axis. The purpose of this review is to examine data from recent interventional studies focusing on probiotics and the gut-brain axis for the treatment of depression, anxiety and schizophrenia.

Recent Findings Probiotics are likely to improve depression but not schizophrenia. Regarding anxiety, there is only one trial which showed an effect of a multispecies probiotic. However, determinants like the duration of treatment, dosage and interactions have not been thoroughly investigated and deserve more scientific attention.

Summary Microbiome-based therapies such as probiotics could be cautiously recommended for depression to enhance beneficial bacteria in the gut and to improve mood through the gut-brain axis.
\end{abstract}

Keywords Probiotics $\cdot$ Microbiota-gut-brain axis $\cdot$ Gut microbiota $\cdot$ Vagal nerve $\cdot$ Psychiatry $\cdot$ Depression $\cdot$ Schizophrenia Anxiety

\section{Introduction}

The gut microbiota is a complex assembly of bacteria, viruses, protozoa, archaea and fungi which inhabit the human gastrointestinal tract (GIT). The number of bacteria in the body slightly exceeds the number of human body cells [1], and not surprisingly, bacteria are essential for a range of physiological processes. Interestingly, cellular organelles such as mitochondria, the adenosine-tri-phosphate (ATP)-generating power plants of the body, are also of bacterial origin and appear to be related to Proteobacteria [2], underlining the central role of bacteria for life, health and disease. The predominant

This article is part of the topical collection on Functional foods

Sabrina Mörk1

sabrina.moerk1@medunigraz.at

1 APC Microbiome Ireland, University College Cork, Cork, Ireland

2 Department of Psychiatry and Psychotherapeutic Medicine, Medical University of Graz, Graz, Austria

3 Department of Psychiatry and Neurobehavioural Science, University College Cork, Cork, Ireland

4 Department of Anatomy and Neuroscience, University College Cork, Cork, Ireland phylotypes in the gut are Firmicutes and Bacteroidetes, but there is a high, finger-print-like individuality of microbial communities, and the terms of a 'healthy gut microbiome' and 'dysbiosis' still remain controversial [3].

There is a complex communication system between the GIT, the micro-organisms which inhabit it and the peripheral and central nervous systems (CNS). This is termed the microbiota-gut-brain axis (MGBA) and constantly transmits and interprets information from the periphery to the brain and back. The exact mechanisms of this communication are still under investigation and involve neural (vagus nerve and enteric nervous system), endocrine (cortisol and hypothalamicpituitary-adrenal (HPA) axis) and immune (cytokine) pathways. It is noteworthy that these pathways are also often found to be altered in the context of psychiatric disorders.

The gut microbiota is a modifiable target with the potential for epigenetic modification [4.] and could therefore be used to treat and ameliorate symptoms of psychiatric disorders. The MGBA can be modified with certain prebiotics (dietary modification/ diets rich in non-digestible fibre), probiotics (living bacteria), antibiotics, synbiotics (combinations of pre- and probiotics), postbiotics (bacterial fermentation products such as short chain fatty acids (SCFAs)) and faecal microbiota transplantation (FMT) [5]. All these approaches could be regarded as potential 
psychobiotics, as they are suggested to improve mental health through their microbiota-modifying properties $[6,7]$.

Probiotics are live organisms, that when administered in adequate amounts, offer health benefits to the host [8]. The treatment of depression and anxiety with probiotics was first suggested in 1910 [9] and then revisited in 2005 [10]. To date, only a limited number of clinical studies have tested the effects of probiotics on the MGBA and their possible efficacy in the treatment of psychiatric disorders. The purpose of this review is to examine the recent literature on the effects of probiotics on the MGBA and to review data from recently published prospective clinical trials which studied probiotics as a treatment for depression, anxiety and schizophrenia.

\section{Search Strategy and Selection Criteria}

We searched PubMed for original research articles, systematic reviews and meta-analysis conducted over the last 5 years (January 2014-December 2019). The following search terms were used: 'probiotics', 'psychobiotics', 'gut-brain axis', 'microbiotagut-brain axis' and combinations with 'depression', 'anxiety', 'social anxiety disorder', 'generalized anxiety disorder', 'schizophrenia', 'inflammation' and 'vagus nerve'. Reference lists of relevant articles were also reviewed to find additional literature.

Human studies were included if they were clinical, randomised controlled trials (RCTs). The study population in these papers must have been clinically diagnosed with either depression, an anxiety disorder or schizophrenia. Relevant questionnaires must have been used to quantify psychiatric symptoms (such as the Beck Depression Inventory (BDI) for depression severity). An intervention of probiotics must have been studied. The following exclusion criteria were relevant: case reports with $n=1$ or a low $n$ number have been excluded; studies investigating subjects with no clinically diagnosed mental health condition or no reported intervention with probiotics. For the creation of Table 2, the population, interventions, comparisons, outcomes and study design (PICOS) criteria were used to summarise the research.

\section{Results}

\section{The Microbiota-Gut-Brain Axis and Its Components}

Gut microbes constantly interact with the brain through a range of pathways, including immune regulation, metabolism of neurotransmitters, SCFAs and vagal afferents [21, 22] (see Fig. 1). Further, the gut microbiota determines stress responsivity by influencing the hypothalamic-pituitary-adrenal axis (HPA axis) [23] and stress cortisol responses can be altered by several probiotics [24, 25]. Elevated stress levels are intertwined with anxiety and depression. The rates of depression and anxiety are disproportionally high in patients with functional gut disorders. Mikocka-Walus et al. found that - by including studies examining either symptoms with validated screening scales (i.e. the Hospital Anxiety and depression scale) or the structured clinical interview for DSM - the pooled mean proportion for anxiety in inflammatory bowel diseases versus healthy controls was 19.1 versus $9.6 \%$. For depression, it was 21.2 versus $13.4 \%$ [26]. Table 1 lists possible mechanisms of psychobiotics on the gut-brain axis.
Fig. 1 Microbiota modulation of the central nervous system (CNS). This figure was created with BioRender.com

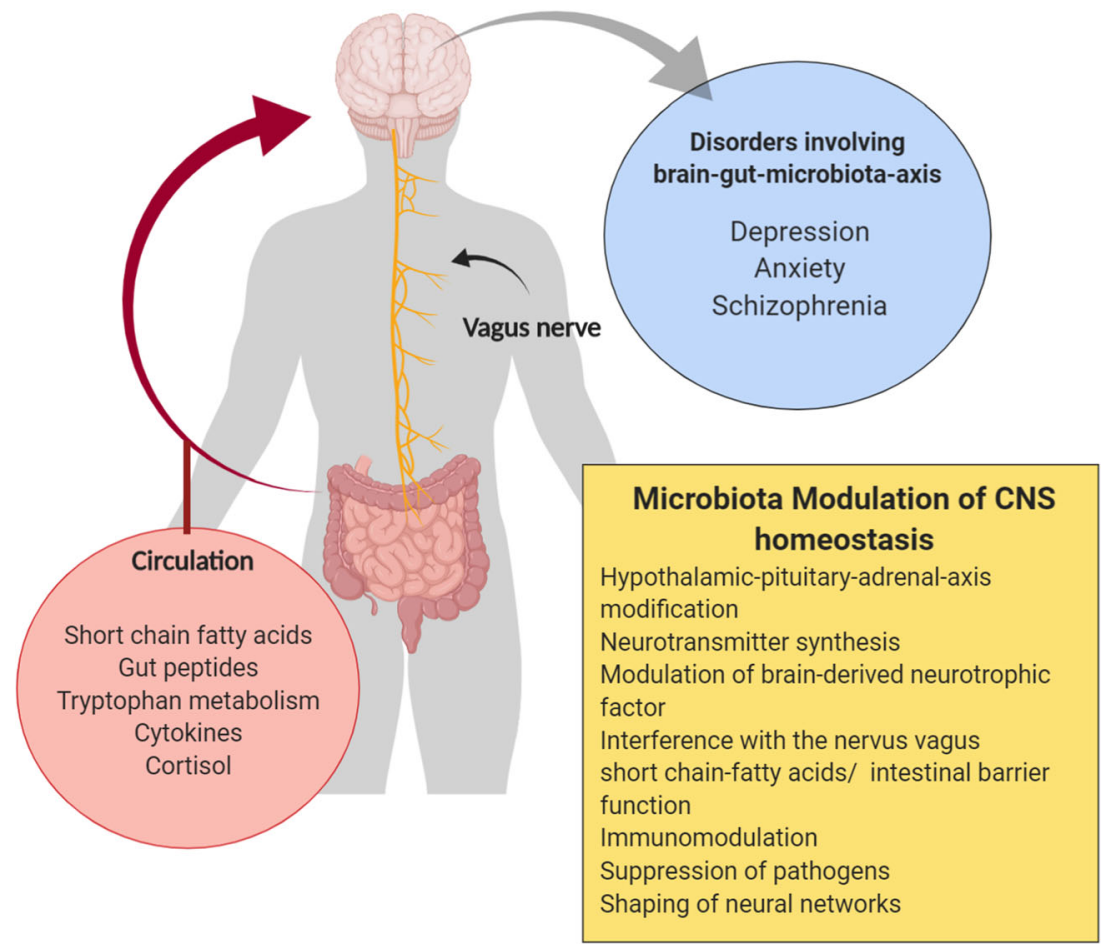


Table 1 Mechanisms of psychobiotic action

Mechanisms of psychobiotic action

Hypothalamic-pituitary-adrenal axis (HPA) modification [27]

Neurotransmitter synthesis (such as gamma

aminobutyric acid, serotonin, dopamine,

noradrenaline, melatonin, histamine and acetylcholine) [28-30]

Modulation of brain-derived neurotrophic factor (BDNF) [31]

Modulation of oxytocin [32]

Interaction with the 10th cranial nerve (nervus vagus) [33]

Postbiotics (such as short chain fatty acids) [34, 35]

Preservation/improvement of the intestinal barrier function [36]

Training of the immune system, immunomodulation [37]

Suppression of pathogens [38]

Shaping of neural networks [39]

\section{Psychobiotics}

Psychobiotics was initially referred to probiotics causing alterations of mood, anxiety and cognitive function [6]. The term 'psychobiotics' now includes all microbiota-targeted interventions such as probiotics and prebiotics that influence bacteria-brain relationships $[7 \bullet]$.

Probiotics, living bacteria with health-improving properties are dosed in 'colony forming units' (CFU) [8, 40]. In most studies, probiotics such as Lactobacillus and Bifidobacteria species [41•] are administered but yeast strains (such as Saccharomyces boulardii) are also used [42]. Probiotics are thought to contribute to a balanced gut environment by suppressing pathogens and interacting with host microbiota. Some bacterial species are not inherently pathogenic as they are found in small abundances in healthy hosts; however, if they become a dominant species in the gut environment, this leads to a disease. Therefore, a diverse environment is of importance and probiotics are thought to contribute to this diversity. Further, they train the immune system and have effects on metabolism and hormone function [43, 44].

One of the major determinants of the gut microbiota composition is prebiotics and diet. Animal- and plant-based diets cause dramatic shifts of the gut microbiota within days [45, 46]. Certain dietary styles, such as the Mediterranean diet, are rich in plantbased foods and fibre that promote the growth of beneficial bacteria [47-49]. Some dietary supplements, such as omega-3 fatty acids, are used in the treatment of depressive disorders [50], but most dietary supplements still lack scientific evidence [51]. Moreover, probiotic food supplements are now extensively tested as an add-on treatment for psychiatric disorders.

\section{Probiotics, Inflammation and the Vagus Nerve}

The inflammatory hypothesis of psychiatric disorders has recently been the centre of attention; however, it is still uncertain where the chronic low-grade inflammation that characterises many psychiatric disorders actually originates [52]. SCFAs such as butyrate are important for gut barrier integrity and affect the CNS by altering the expression of brain-derived neurotrophic factor (BDNF). These SCFAs have been found to be of importance in psychiatric disorders; for example, they were found to be lower in depression [53]. SCFAs are vital for gut barrier function. A disruption in gut barrier integrity could lead to the translocation of bacteria and bacterial antigens (such as lipopolysaccharides) into the blood stream causing chronic low-grade inflammation [54].

To maintain homeostasis, the CNS responds constantly to environmental cues transmitted by the vagus nerve, which is one of the main players in MGBA communication. Peripheral cytokine production triggers the vagal anti-inflammatory reflex leading to production of acetylcholine which thereby prevents tissue damage by excessive cytokine release [55]. Recent research pointed out alterations of gut microbiota [56-60] as well as vagal tone in depressed individuals [61], patients with anxiety disorders [62] and schizophrenia [63]. Some probiotics, such as Bifidobacterium signal to the brain via vagal pathways [64, 65]. When the vagal nerve is cut, some probiotics no longer show effects on brain and behaviour [33, 66, 67].

\section{Probiotics to Modify the Gut-Brain Axis (Human Studies)}

The gut microbiota impacts brain function, and an array of clinical studies provide us with insights into possible mechanisms. The clinical implications of probiotic use are currently under investigation for psychiatric indications. Recent trials of probiotic treatments yielded inconsistent results. Following a search with the relevant keywords, nine RCTs matched the inclusion criteria. Four RCTs included patients with schizophrenia, five RCTs included patients with depression and one RCT included patients with an anxiety disorder (generalised anxiety disorder). Table 2 gives an overview of RCTs published over the past 5 years (2014-2019), focusing on probiotics for the treatment of depression, anxiety and schizophrenia.

\section{Probiotics and Depression}

Major depression is among the most prevalent disorders worldwide and therefore is of utmost importance in the context of health policy [68]. Patients with depression show significant differences in gut microbiota composition in comparison with those without depression [56-60, 69]. When rats are colonised with faecal matter from patients with depression, they exhibit depressive-like symptoms [58]. However, there is no specific 'dysbiosis' signature found in depression. A variety of studies have investigated probiotic effects on mood. Most of them have been done in healthy populations or in participants without an adequately diagnosed depressive disorder. To date, several metaanalyses support the use of probiotics to improve mood [70-73]. 

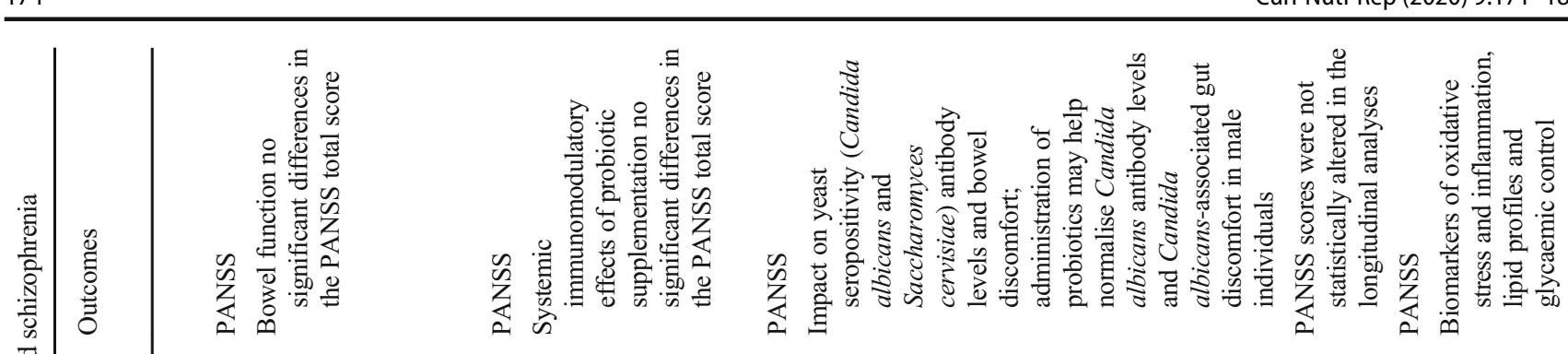

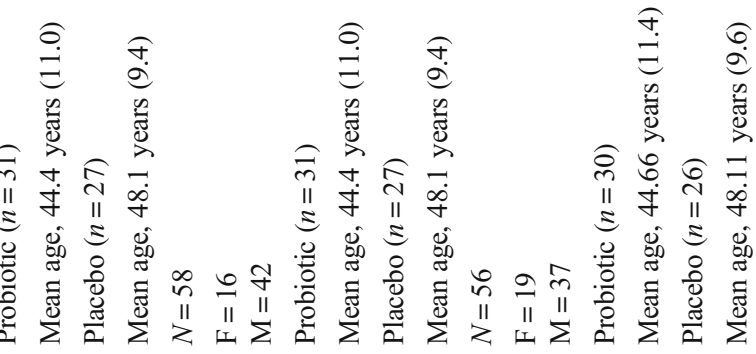

in

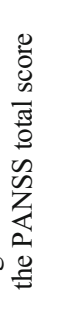

$\Xi$

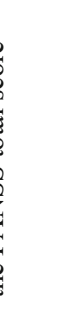

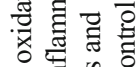

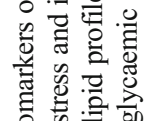
๓
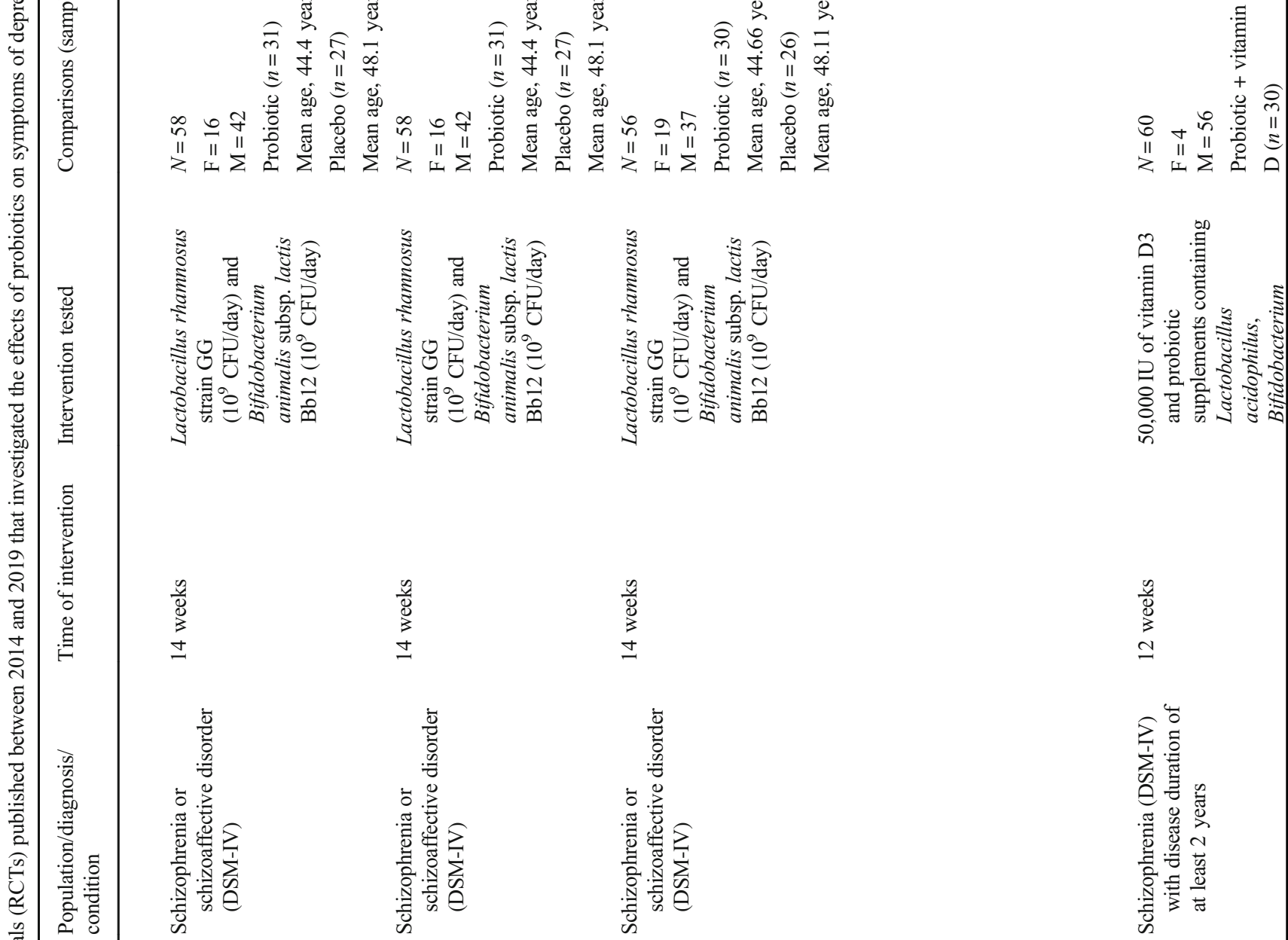

$n$
$\frac{y}{0}$
$\vdots$
$\vdots$
$\Xi$

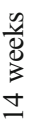

$y$
$\frac{y}{0}$
$\vdots$
$\simeq$
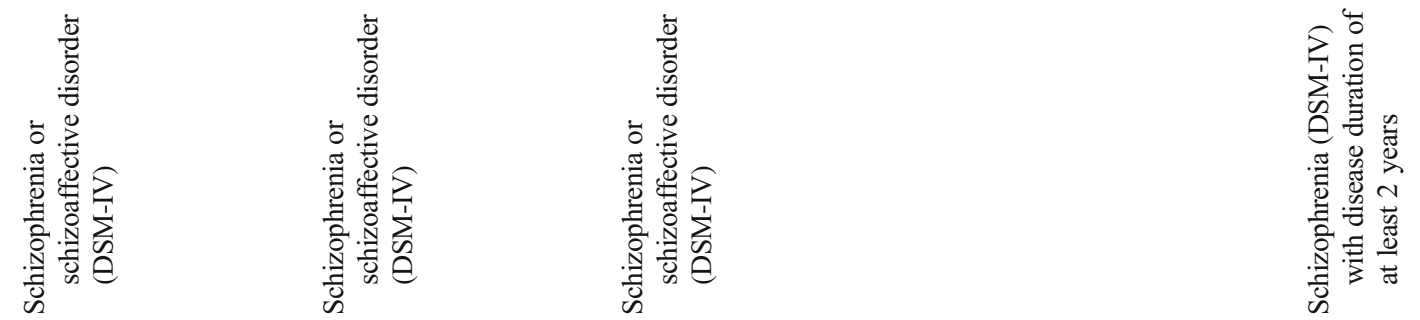

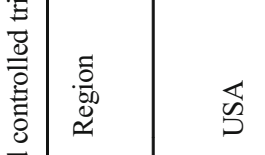

岕

店

콥

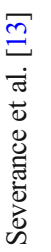

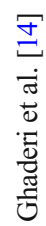




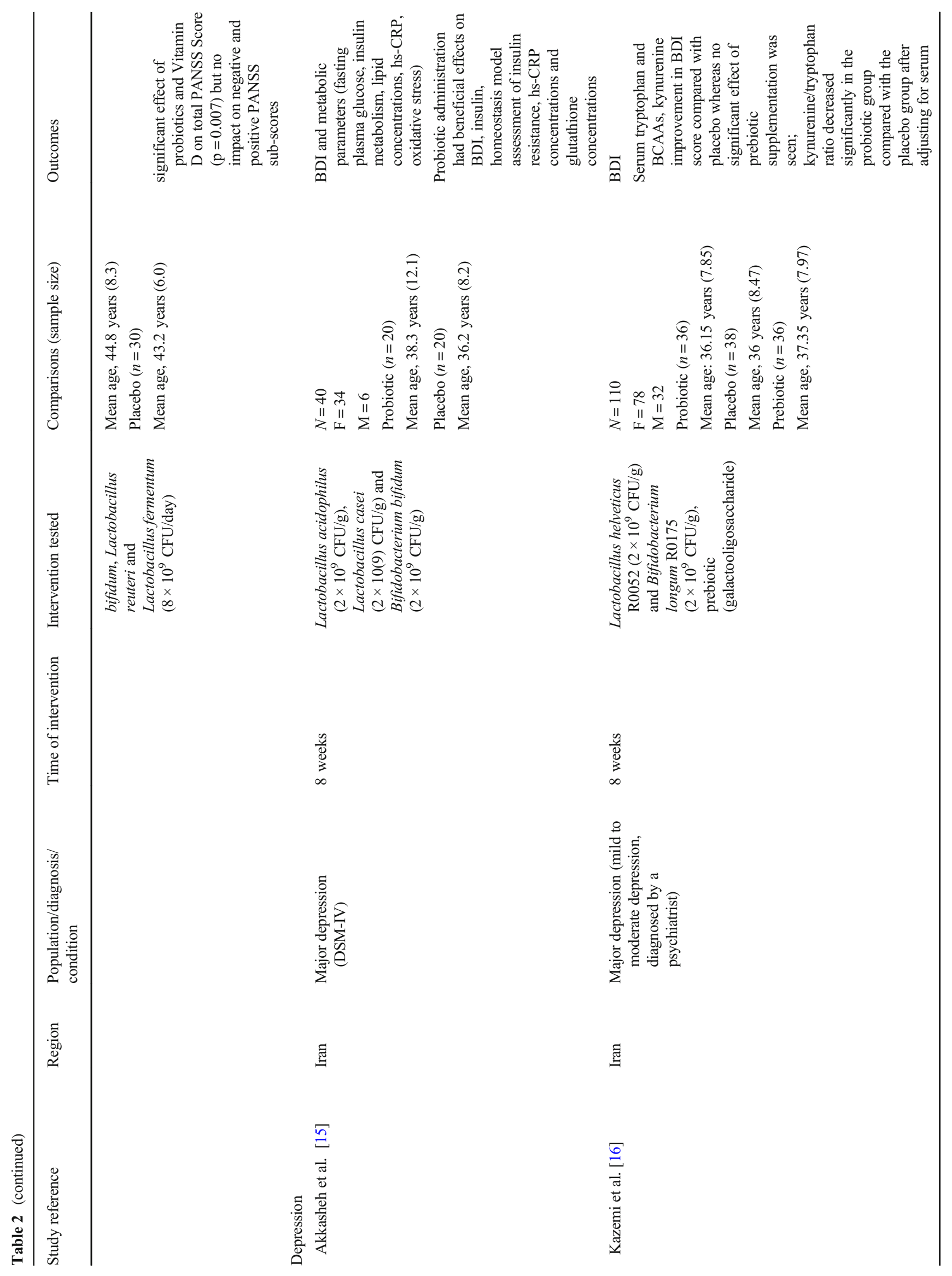




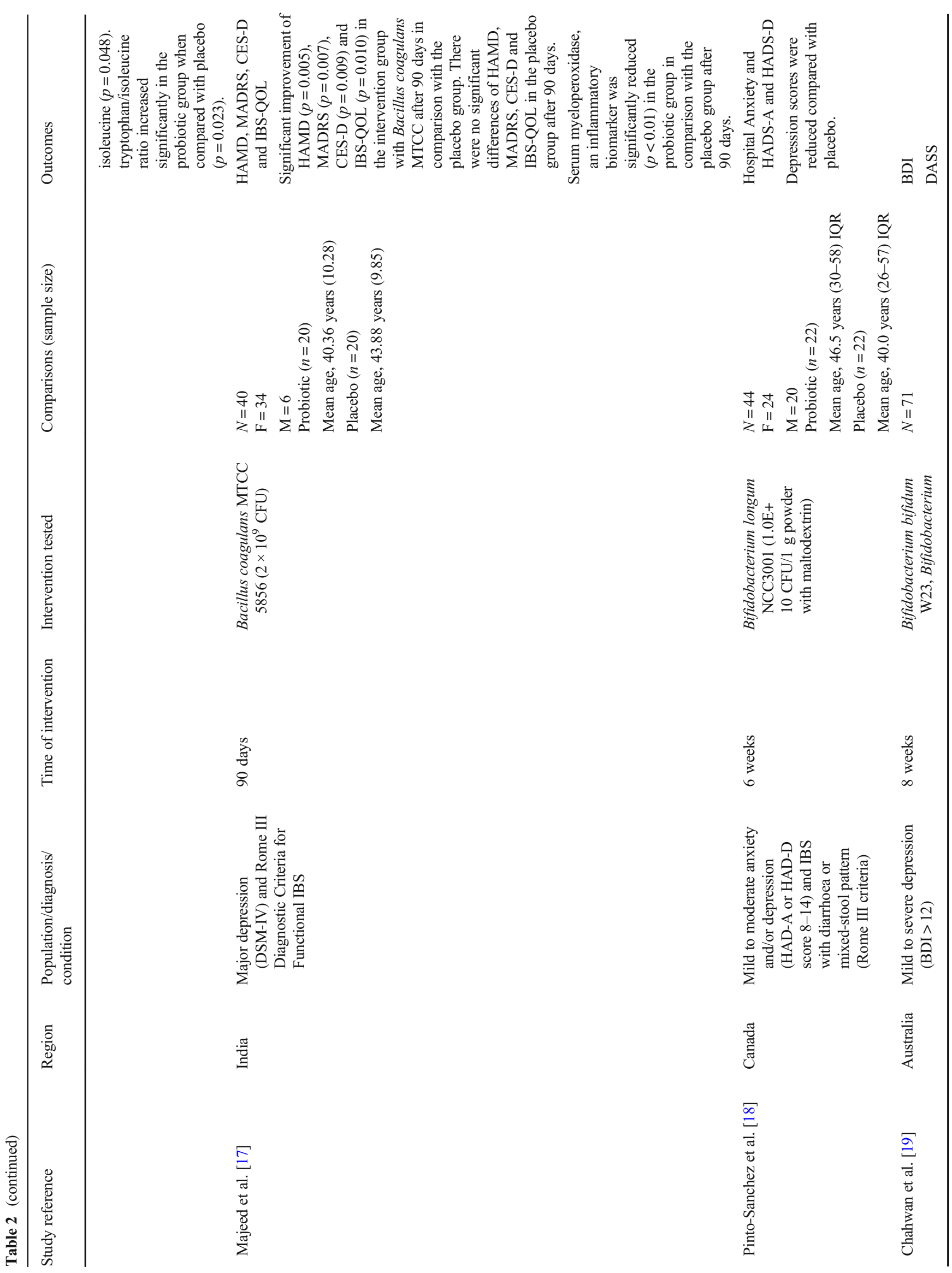




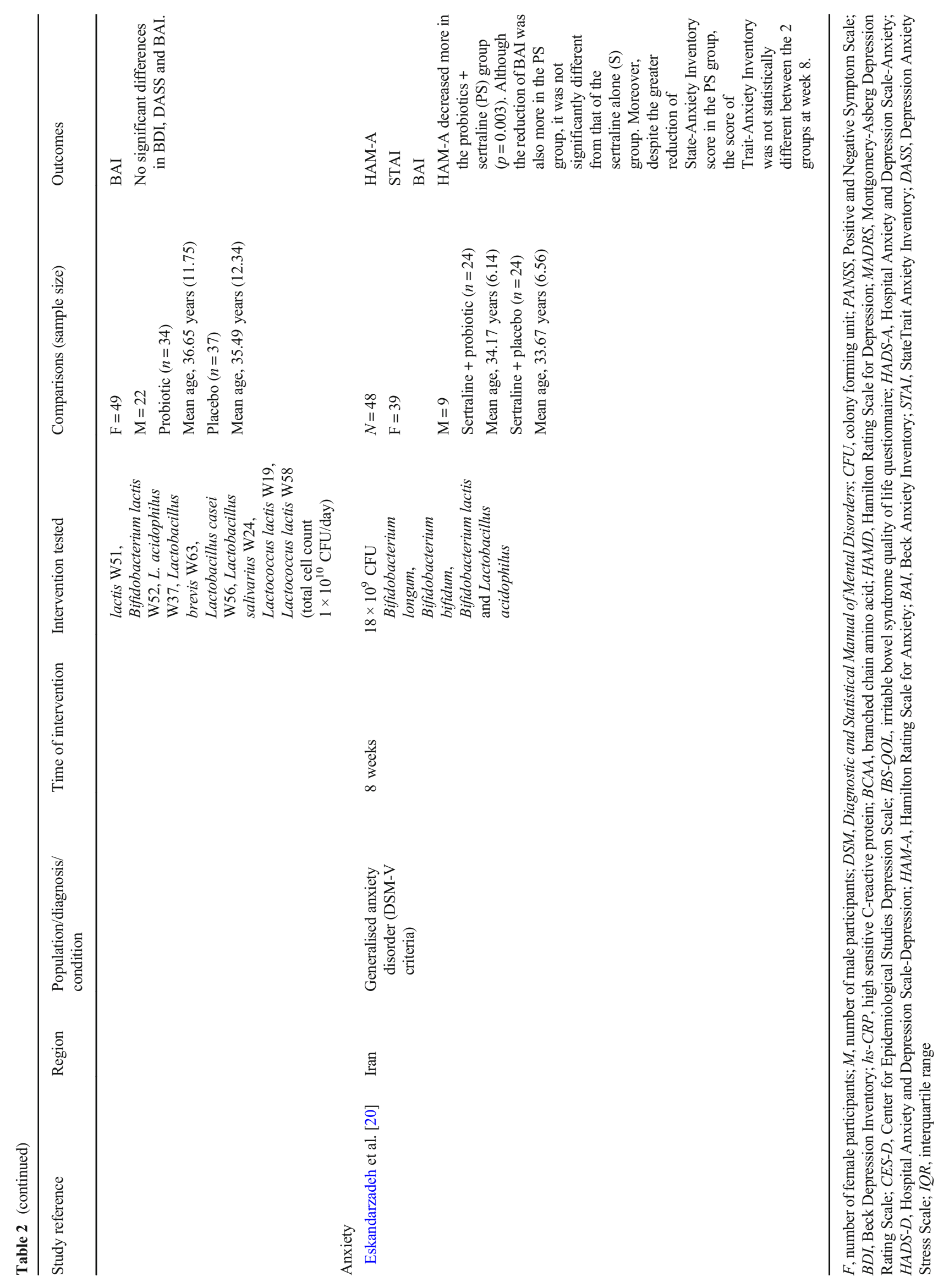


However, mood effects are only significant in participants exhibiting symptoms of depression [72].

Currently, there are five probiotic RCTs using predominantly Lactobacillus and Bifidobacterium species to treat depression (see Table 1). Akkaseh et al. included 40 participants with major depressive disorder (MDD) in the probiotic RCT [15]. After 8 weeks, the 20 patients in the active intervention group showed significantly decreased BDI scores in comparison with the placebo group.

Another RCT from Kazemi et al. included 110 participants, with 36 receiving a probiotic, 38 receiving placebo and 35 receiving a prebiotic [16]. After 8 weeks of supplementation, the probiotic group showed a significant reduction of the BDI score in comparison with the placebo and probiotic supplementation group.

Majeed et al. included 40 patients with a co-diagnosis of MDD and irritable bowel syndrome (IBS). Twenty were allocated to the probiotic group and twenty to the placebo group for a 90-day intervention. After the intervention, the probiotic group showed a significant improvement on the depression scales (Hamilton Rating Scale for Depression (HAMD), Montgomery-Åsberg Depression Rating Scale (MADRS), Center for Epidemiologic Studies Depression Scale (CES-D)). However, in this study, clear conclusions regarding patients with MDD cannot be made, because of the co-diagnosis of IBS.

A significant reduction of depression scores but not anxiety scores was found in the RCT of Pinto-Sanchez et al. [18], after a 6-week treatment of 22 patients receiving Bifidobacterium longum in comparison with 22 patients receiving placebo.

The latest study of Chahwan did not find a significant effect on depressive symptoms following an 8 week intervention with a multi-strain probiotic [19].

However, all these studies lacked gut microbiota profiling of patients before and after probiotic use. Moreover, these studies show some discrepancies regarding strains and duration of treatment (reaching from 6 to 13 weeks). Three of five studies used combinations of Lactobacillus and Bifidobacterium species [15, 16, 19], while two of five studies used single strains such as Bifidobacterium longum [18] and Bacillus coagulans [17]. Due to the paucity of studies, direct conclusions on the optimal strain combinations and duration of treatment cannot be drawn. However, long-term probiotic supplementation may have some merit as probiotics cannot be detected in stool 1-4 weeks after the consumption is stopped [74]. For example, in the study of Pinto-Sanchez, depression scores were still significantly better compared with baseline in the follow-up ( 4 weeks after the end of the probiotic intervention), but the depression scores were rising again [18].

\section{Probiotics and Anxiety}

There have been multiple studies examining the effects of probiotics on anxiety symptoms in other diseases such as
IBS (for a review and meta-analysis, see, [75]). In animal studies, stress, HPA axis response and anxiety-related behaviour were affected after probiotic intake [76, 77]; however, results were often inconsistent [75].

To our best knowledge there is only one single publication reporting RCT data of a probiotic to treat patients with a diagnosed anxiety disorder (generalised anxiety disorder (DSM-V criteria)) [20•]. This small Iranian RCT tested the impact of an 8-week intervention with a multi-strain probiotic containing Bifidobacterium longum, Bifidobacterium bifidum, Bifidobacterium lactis and Lactobacillus acidophilus. Twenty-four patients were assigned to the probiotic intervention group and twentyfour to the control group. Probiotic and placebo capsules were given as an add-on therapy as both the control and probiotic intervention group received a baseline selective serotonin reuptake inhibitor (SSRI) therapy with sertraline. They used the Hamilton Rating Scale for Anxiety (HAM-A), the Beck Anxiety Inventory (BAI) and the State Trait Anxiety Inventory (STAI) to quantify anxiety symptoms before and after the probiotic intervention. After 8 weeks, there was a significant reduction in the HAM-A score in the group receiving probiotics and sertraline in comparison with the sertraline plus placebo group. However, the BAI score was not significantly different. After 8 weeks, only state anxiety was different in the group receiving sertraline plus probiotic but not trait anxiety. In relation to biological markers, the researchers measured ACTH and serum cortisol levels. These parameters did not significantly change in either the intervention or the control group.

Unfortunately, there have been no other interventional studies in people with clinically relevant anxiety disorders. Further research in this area should be done given this small but encouraging trial and the ever-expanding literature outlining promising preclinical results.

\section{Probiotics and Schizophrenia}

Schizophrenia is mainly a heritable disorder; however, many researchers assume a possible aetiological role of the gut microbiome through epigenetic modulation (i.e. diet and exposure to infectious agents), influence on the immune system and neuroinflammation $[78,79]$. Interestingly, many of the genetic loci related to schizophrenia are known to modulate the immune system and inflammation [80]. Moreover, central neurotransmitter changes were found in mice after receiving a FMT from patients with schizophrenia [81].

For schizophrenia, there are three RCTs, which were already systematically reviewed by $\mathrm{Ng}$ et al. They did not find a significant difference in schizophrenia symptoms between probiotic and placebo groups postintervention when applying a per-protocol analysis and a fixed effects model [82]. 
All of these three studies were from the same research group. They included patients with schizophrenia or schizoaffective disorder and tested the same intervention (multistrain-probiotic containing Lactobacillus rhamnosus and Bifidobacterium animalis subsp. lactis). With this probiotic no significant effects on the Positive and Negative Symptom Scale (PANSS) total score ( $p=$ 0.25 ) could be found after 14 weeks of intervention in all three studies. However, Dickerson et al. reported a reduced risk for severe bowel problems in patients with moderate to severe schizophrenia symptoms after treatment with the probiotic supplement $(p=0.003)$ [11]. Tomasik et al. found systemic immunomodulatory effects (via cytokine modulation) of probiotic supplementation (reduction of the acute-phase reactant von Willebrand factor, $p=0.047)$. Another RCT by Severance et al. showed an inverse link of $C$. albicans antibody level with GI symptoms in patients with schizophrenia. The most recent study tested a probiotic supplement in combination with vitamin D3 [14]. Ghaderi et al. showed a significant effect of a 12week intervention on the PANSS score $(p=0.007)$; however, there was no impact of the intervention on PANSS subscores.

\section{Conclusions-Probiotics as Modifiers of the Gut-Brain Axis}

In this review, we summarise important clinical findings regarding the MGBA and results from recent RCTs focusing on probiotic interventions for psychiatric disorders. Probiotics appear to have an impact on symptoms of depression but not schizophrenia. As there is only one RCT so far using a probiotic as an adjunctive treatment for anxiety, no firm conclusions can be drawn.

The MGBA provides the field of psychiatry a new paradigm for the treatment of mental illness. Despite receiving up-to-date, evidence-based, multimodal treatments, many psychiatric patients continue to experience distressing symptoms. Even with conflicting clinical results, probiotic use is greatly popularised in the media and probiotics belong to the most commonly consumed food supplements [83].

It should be mentioned that, although modulation of the MGBA with probiotics appear promising as a therapeutic strategy for mental illness, several challenges remain. First, RCTs published to date display comparably small sample sizes and methodological heterogeneity. Many studies also only use self-reported parameters of symptomatology without a sufficient assessment of subjects or to confirm a clinical diagnosis and screen for comorbidities. Secondly, probiotics may not work in the same way for every patient. For example, a recent study from Washington State University has shown that under certain conditions, ingested probiotics could evolve and adapt in either a positive or a negative way according to the given environment in the gut. As living organisms, probiotics are subjected to natural selection. For example, the probiotic bacterium Escherichia coli Nissle was found to enhance mucin utilisation in lowdiversity environments which could damage the intestinal lining [84].

Another important contributor to the high variability of results in probiotic studies is the variety of studied strains and strain combinations. For example, different strains of the same species have demonstrated opposing effects in relation to psychological symptoms: while Lactobacillus rhamnosus (strain JB-1) did not affect mood or anxiety levels in healthy men [85], Lactobacillus casei (strain Shirota) improved mood in participants with low baseline mood scores [86].

This underlines the necessity of combining probiotics with a diet containing an adequate amount of micro- and macronutrients to promote favourable development of the gut flora. Notably, individuals suffering from psychiatric illness, and especially individuals with schizophrenia, show poor dietary patterns [87]. Furthermore, the gut microbiome can also be altered by certain psychotropic medications, which should be taken into account [88 ${ }^{\bullet}$. In particular, antidepressants and antipsychotics could alter the gut microbiota of the host [89-91].

A species and strain-sensitive assessment of participants evaluated mucosal colonisation after consumption of 11 probiotic strains and found that $40 \%$ of the tested individuals showed a near-total colonisation resistance after probiotic ingestion and the degree of mucosal association could be predicted by baseline host and microbiome factors [92]. In light of this, an unresolved issue is whether gut colonisation by probiotics is stable or merely a transient event [92]. Further research should focus on individual, personalised approaches including a targeted therapy with pre- and probiotics according to the gut environment of the individual. This therapy should also take environmental factors (diet, fluid intake, age, gender, comorbidities) into account.

Against this background, the area of nutrition and gut health will likely become an important component in the biopsychosocial treatment model in psychiatry. The evolving field of nutritional psychiatry should therefore be integrated in clinical practice to treat and prevent psychiatric disorders as well as metabolic comorbidities [93].

In summary, probiotics could be used as an add-on treatment for some psychiatric indications such as depression; however, as effect sizes are low, they are unlikely to substitute psychopharmacological approaches in the future. Especially for anxiety disorders, the evidence is very weak, and there is still a huge research gap which needs to be filled in the years to come. 
Funding Information Open access funding provided by Medical University of Graz. The APC Microbiome Ireland is a research institute funded by Science Foundation Ireland (SFI). J.F.C and T.G.D are supported by SFI (Grant Nos. SFI/12/RC/2273).

\section{Compliance with Ethical Standards}

Conflict of Interest All authors declare that they have no conflict of interest.

Human and Animal Rights and Informed Consent This article does not contain any studies with human or animal subjects performed by the authors.

Open Access This article is licensed under a Creative Commons Attribution 4.0 International License, which permits use, sharing, adaptation, distribution and reproduction in any medium or format, as long as you give appropriate credit to the original author(s) and the source, provide a link to the Creative Commons licence, and indicate if changes were made. The images or other third party material in this article are included in the article's Creative Commons licence, unless indicated otherwise in a credit line to the material. If material is not included in the article's Creative Commons licence and your intended use is not permitted by statutory regulation or exceeds the permitted use, you will need to obtain permission directly from the copyright holder. To view a copy of this licence, visit http://creativecommons.org/licenses/by/4.0/.

\section{References}

Papers of particular interest, published recently, have been highlighted as:

- Of importance

1. Sender R, Fuchs S, Milo R. Revised estimates for the number of human and bacteria cells in the body. PLoS Biology. 2016;14(8): e1002533-e. https://doi.org/10.1371/journal.pbio.1002533.

2. Roger AJ, Muñoz-Gómez SA, Kamikawa R. The origin and diversification of mitochondria. Curr Biol. 2017;27(21):R1177-R92.

3. Moloney RD, Desbonnet L, Clarke G, Dinan TG, Cryan JF. The microbiome: stress, health and disease. Mamm Genome. 2014;25(1-2):49-74. https://doi.org/10.1007/s00335-013-9488-5.

4. Miro-Blanch J, Yanes O. Epigenetic regulation at the interplay between gut microbiota and host metabolism. Front Genet. 2019;10 This manuscript explores the importance of epigenetic regulation of host tissues by the metabolic activity of gut microbiota in response to changes in diet.

5. Zmora N, Soffer E, Elinav E. Transforming medicine with the microbiome. Science Translational Medicine. 2019;11(477): eaaw1815.

6. Dinan TG, Stanton C, Cryan JF. Psychobiotics: a novel class of psychotropic. Biol Psychiatry. 2013;74(10):720-6. https://doi.org/ 10.1016/j.biopsych.2013.05.001.

7. Sarkar A, Lehto SM, Harty S, Dinan TG, Cryan JF, PWJ B. Psychobiotics and the manipulation of bacteria-gut-brain signals. Trends Neurosci. 2016;39(11):763-81. https://doi.org/10.1016/j. tins.2016.09.002 This review outlines the new definition of a psychobiotic, including not only probiotics but also prebiotics which influence bacteria-brain relationships.

8. Butel MJ. Probiotics, gut microbiota and health. Medecine et maladies infectieuses. 2014;44(1):1-8. https://doi.org/10.1016/j. medmal.2013.10.002.
9. Phillips JGP. The treatment of melancholia by the lactic acid bacillus. J Ment Sci. 1910;56(234):422-30.

10. Logan AC, Katzman M. Major depressive disorder: probiotics may be an adjuvant therapy. Med Hypotheses. 2005;64(3):533-8.

11. Dickerson FB, Stallings C, Origoni A, Katsafanas E, Savage CL, Schweinfurth LA, et al. Effect of probiotic supplementation on schizophrenia symptoms and association with gastrointestinal functioning: a randomized, placebo-controlled trial. Prim Care Companion CNS Disord. 2014;16(1):PCC.13m01579. https://doi. org/10.4088/PCC.13m01579.

12. Tomasik J, Yolken RH, Bahn S, Dickerson FB. Immunomodulatory effects of probiotic supplementation in schizophrenia patients: a randomized. Placebo-Controlled Trial Biomark Insights. 2015;10(1):47-54. https://doi.org/10.4137/BMI.S22007.

13. Severance EG, Gressitt KL, Stallings CR, Katsafanas E, Schweinfurth LA, Savage CL, et al. Probiotic normalization of Candida albicans in schizophrenia: a randomized, placebo-controlled, longitudinal pilot study. Brain Behav Immun. 2017;62:41-5.

14. Ghaderi A, Banafshe HR, Mirhosseini N, Moradi M, Karimi MA, Mehrzad F, et al. Clinical and metabolic response to vitamin D plus probiotic in schizophrenia patients. BMC Psychiatry. 2019;19(1): 77. https://doi.org/10.1186/s12888-019-2059-X.

15. Akkasheh G, Kashani-Poor Z, Tajabadi-Ebrahimi M, Jafari P, Akbari H, Taghizadeh M, et al. Clinical and metabolic response to probiotic administration in patients with major depressive disorder: a randomized, double-blind, placebo-controlled trial. Nutrition (Burbank, Los Angeles County, Calif). 2016;32(3):315-20. https:// doi.org/10.1016/j.nut.2015.09.003.

16. Kazemi A, Noorbala AA, Azam K, Eskandari MH, Djafarian K. Effect of probiotic and prebiotic vs placebo on psychological outcomes in patients with major depressive disorder: a randomized clinical trial. Clinical Nutrition (Edinburgh, Scotland). 2019;38(2): 522-8. https://doi.org/10.1016/j.clnu.2018.04.010.

17. Majeed M, Nagabhushanam K, Arumugam S, Majeed S, Ali F. Bacillus coagulans MTCC 5856 for the management of major depression with irritable bowel syndrome: a randomised, doubleblind, placebo controlled, multi-centre, pilot clinical study. Food Nutr Res. 2018;62. https://doi.org/10.29219/fnr.v62.1218.

18. Pinto-Sanchez MI, Hall GB, Ghajar K, Nardelli A, Bolino C, Lau JT, et al. Probiotic Bifidobacterium longum NCC3001 reduces depression scores and alters brain activity: a pilot study in patients with irritable bowel syndrome. Gastroenterology. 2017;153(2): 448-59.e8. https://doi.org/10.1053/j.gastro.2017.05.003.

19. Chahwan B, Kwan S, Isik A, van Hemert S, Burke C, Roberts L. Gut feelings: a randomised, triple-blind, placebo-controlled trial of probiotics for depressive symptoms. J Affect Disord. 2019;253:317-26.

20. Eskandarzadeh S, Effatpanah M, Khosravi-Darani K, Askari R, Hosseini AF, Reisian M, et al. Efficacy of a multispecies probiotic as adjunctive therapy in generalized anxiety disorder: a double blind, randomized, placebo-controlled trial. Nutritional Neuroscience. 2019:1-7. https://doi.org/10.1080/1028415x.2019. 1598669 This is the first double-blind randomized control trial of a multispecies probiotic for anxiety disorders.

21. Wang Y, Yuan X, Kang Y, Song X. Tryptophan-kynurenine pathway as a novel link between gut microbiota and schizophrenia: a review. Trop J Pharm Res. 2019;18(5):897-905. https://doi.org/10.4314/tjpr.v18i4.30.

22. Yuan X, Kang Y, Zhuo C, Huang X-F, Song X. The gut microbiota promotes the pathogenesis of schizophrenia via multiple pathways. Biochem Biophys Res Commun. 2019;512(2):373-80.

23. Rea K, Dinan TG, Cryan JF. The microbiome: a key regulator of stress and neuroinflammation. Neurobiology of Stress. 2016;4:2333. https://doi.org/10.1016/j.ynstr.2016.03.001.

24. Takada M, Nishida K, Kataoka-Kato A, Gondo Y, Ishikawa H, Suda K, et al. Probiotic Lactobacillus casei strain Shirota relieves stress-associated symptoms by modulating the gut-brain interaction in human and animal models. Neurogastroenterology and Motility: 
the Official Journal of the European Gastrointestinal Motility Society. 2016;28(7):1027-36. https://doi.org/10.1111/nmo.12804.

25. Allen AP, Hutch W, Borre YE, Kennedy PJ, Temko A, Boylan G, et al. Bifidobacterium longum 1714 as a translational psychobiotic: modulation of stress, electrophysiology and neurocognition in healthy volunteers. Transl Psychiatry. 2016;6(11):e939. https:// doi.org/10.1038/tp.2016.191.

26. Mikocka-Walus A, Knowles SR, Keefer L, Graff L. Controversies revisited: a systematic review of the comorbidity of depression and anxiety with inflammatory bowel diseases. Inflamm Bowel Dis. 2016;22(3):752-62.

27. de Weerth C. Do bacteria shape our development? Crosstalk between intestinal microbiota and HPA axis. Neurosci Biobehav Rev. 2017;83:458-71.

28. Daliri E, Oh D, Lee B. Psychobiotics; a promise for neurodevelopmental therapy. J Probiotics Health. 2016;4:1e4.

29. Sampson TR, Mazmanian SK. Control of brain development, function, and behavior by the microbiome. Cell Host Microbe. 2015;17(5):565-76.

30. Roshchina V. Evolutionary considerations of neurotransmitters in microbial, plant, and animal cells. In: Lyte M, Freestone P, editors. Microbial endocrinology: interkingdom signaling in infectious disease and health. New York: Springer; 2010. p. 17-52.

31. Ranuh R, Athiyyah AF, Darma A, Risky VP, Riawan W, Surono IS, et al. Effect of the probiotic Lactobacillus plantarum IS-10506 on BDNF and 5HT stimulation: role of intestinal microbiota on the gut-brain axis. Iran J Microbiol. 2019;11(2):145-50.

32. Evans SJ, Bassis CM, Hein R, Assari S, Flowers SA, Kelly MB, et al. The gut microbiome composition associates with bipolar disorder and illness severity. J Psychiatr Res. 2017;87:23-9. https:// doi.org/10.1016/j.jpsychires.2016.12.007.

33. Fülling C, Dinan TG, Cryan JF. Gut microbe to brain signaling: what happens in vagus. Neuron. 2019;101(6):998-1002.

34. LeBlanc JG, Chain F, Martín R, Bermúdez-Humarán LG, Courau S, Langella P. Beneficial effects on host energy metabolism of short-chain fatty acids and vitamins produced by commensal and probiotic bacteria. Microb Cell Factories. 2017;16(1):79.

35. Bourassa MW, Alim I, Bultman SJ, Ratan RR. Butyrate, neuroepigenetics and the gut microbiome: can a high fiber diet improve brain health? Neurosci Lett. 2016;625:56-63. https://doi. org/10.1016/j.neulet.2016.02.009.

36. Bron PA, Kleerebezem M, Brummer R-J, Cani PD, Mercenier A, MacDonald TT, et al. Can probiotics modulate human disease by impacting intestinal barrier function? Br J Nutr. 2017;117(1):93-107.

37. Frei R, Akdis M, O’Mahony L. Prebiotics, probiotics, synbiotics, and the immune system: experimental data and clinical evidence. Curr Opin Gastroenterol. 2015;31(2):153-8.

38. Suez J, Zmora N, Segal E, Elinav E. The pros, cons, and many unknowns of probiotics. Nat Med. 2019;25(5):716-29. https://doi. org/10.1038/s41591-019-0439-x.

39. Möhle L, Mattei D, Heimesaat MM, Bereswill S, Fischer A, Alutis $\mathrm{M}$, et al. Ly6Chi monocytes provide a link between antibioticinduced changes in gut microbiota and adult hippocampal neurogenesis. Cell Rep. 2016;15(9):1945-56.

40. Fuller R. Probiotics: the scientific basis: Springer Science \& Business Media; 2012.

41. Butler MI, Mörkl S, Sandhu KV, Cryan JF, Dinan TG. The gut microbiome and mental health: what should we tell our patients? [Le microbiote Intestinal et la Santé Mentale: que Devrions-Nous dire à nos Patients?]. Can J Psychiatry. 2019:0706743719874168 This review highlights microbiota alterations in patients with psychiatric disorders.

42. Surawicz CM, Elmer GW, Speelman P, McFarland LV, Chinn J, Van Belle G. Prevention of antibiotic-associated diarrhea by Saccharomyces boulardii: a prospective study. Gastroenterology. 1989;96(4):981-8
43. El Aidy S, Dinan TG, Cryan JF. Gut microbiota: the conductor in the Orchestra of Immune-Neuroendocrine Communication. Clin Ther. 2015;37(5):954-67. https://doi.org/10.1016/j.clinthera.2015.03.002.

44. Patterson E, Ryan PM, Cryan JF, Dinan TG, Ross RP, Fitzgerald GF, et al. Gut microbiota, obesity and diabetes. Postgrad Med J. 2016;92(1087):286-300. https://doi.org/10.1136/postgradmedj2015-133285.

45. David LA, Maurice CF, Carmody RN, Gootenberg DB, Button JE, Wolfe BE, et al. Diet rapidly and reproducibly alters the human gut microbiome. Nature. 2014;505(7484):559-63. https://doi.org/10. 1038/nature12820.

46. De Filippis F, Pellegrini N, Vannini L, Jeffery IB, La Storia A, Laghi L, et al. High-level adherence to a Mediterranean diet beneficially impacts the gut microbiota and associated metabolome. Gut. 2016;65(11):1812-21.

47. Gibson GR, Roberfroid MB. Dietary modulation of the human colonic microbiota: introducing the concept of prebiotics. J Nutr. 1995;125(6): 1401-12.

48. Liu RT, Walsh RF, Sheehan AE. Prebiotics and probiotics for depression and anxiety: a systematic review and meta-analysis of controlled clinical trials. Neurosci Biobehav Rev. 2019;102:13-23.

49. Bailey MA, Holscher HD. Microbiome-mediated effects of the Mediterranean diet on inflammation. Adv Nutr. 2018;9(3):193-206

This recent systematic review highlights the role of pro-and prebiotics in the treatment of depression and anxiety. While prebiotics did not differ from placebo, probiotics exhibited small significant effects for the treatment of depression and anxiety.

50. Guu T-W, Mischoulon D, Sarris J, Hibbeln J, McNamara RK, Hamazaki $\mathrm{K}$, et al. International Society for Nutritional Psychiatry Research practice guidelines for omega-3 fatty acids in the treatment of major depressive disorder. Psychother Psychosom. 2019;88(5):263-73.

51. Firth J, Teasdale SB, Allott K, Siskind D, Marx W, Cotter J, et al. The efficacy and safety of nutrient supplements in the treatment of mental disorders: a meta-review of meta-analyses of randomized controlled trials. World Psychiatry. 2019;18(3):308-24.

52. Bauer ME, Teixeira AL. Inflammation in psychiatric disorders: what comes first? Ann N Y Acad Sci. 2019;1437(1):57-67. https://doi.org/10.1111/nyas.13712.

53. Lv F, Chen S, Wang L, Jiang R, Tian H, Li J, et al. The role of microbiota in the pathogenesis of schizophrenia and major depressive disorder and the possibility of targeting microbiota as a treatment option. Oncotarget. 2017;8(59):100899-907.

54. Foster JA, Rinaman L, Cryan JF. Stress \& the gut-brain axis: regulation by the microbiome. Neurobiol Stress. 2017;7:124-36. https://doi.org/10.1016/j.ynstr.2017.03.001.

55. Rosas-Ballina M, Olofsson PS, Ochani M, Valdes-Ferrer SI, Levine YA, Reardon C, et al. Acetylcholine-synthesizing T cells relay neural signals in a vagus nerve circuit. Science (New York, NY). 2011;334(6052):98-101. https://doi.org/10.1126/science.1209985.

56. Naseribafrouei A, Hestad K, Avershina E, Sekelja M, Linlokken A, Wilson R, et al. Correlation between the human fecal microbiota and depression. Neurogastroenterology and Motility: the Official Journal of the European Gastrointestinal Motility Society. 2014;26(8):1155-62. https://doi.org/10.1111/nmo.12378.

57. Jiang H, Ling Z, Zhang Y, Mao H, Ma Z, Yin Y, et al. Altered fecal microbiota composition in patients with major depressive disorder. Brain Behav Immun. 2015;48:186-94. https://doi.org/10.1016/j. bbi.2015.03.016.

58. Kelly JR, Borre Y, O'Brien C, Patterson E, El Aidy S, Deane J, et al. Transferring the blues: depression-associated gut microbiota induces neurobehavioural changes in the rat. J Psychiatr Res. 2016;82:109-18. https://doi.org/10.1016/j.jpsychires.2016.07.019.

59. Zheng P, Zeng B, Zhou C, Liu M, Fang Z, Xu X, et al. Gut microbiome remodeling induces depressive-like behaviors through a pathway mediated by the host's metabolism. Mol Psychiatry. 2016;21(6):786-96. https://doi.org/10.1038/mp.2016.44. 
60. Lin P, Ding B, Feng C, Yin S, Zhang T, Qi X, et al. Prevotella and Klebsiella proportions in fecal microbial communities are potential characteristic parameters for patients with major depressive disorder. J Affect Disord. 2017;207:300 - 4. https://doi.org/10.1016/j.jad. 2016.09.051.

61. Kemp AH, Quintana DS, Felmingham KL, Matthews S, Jelinek HF. Depression, comorbid anxiety disorders, and heart rate variability in physically healthy, unmedicated patients: implications for cardiovascular risk. PLoS One. 2012;7(2):e30777. https://doi.org/ 10.1371/journal.pone.0030777.

62. Chalmers JA, Quintana DS, Abbott MJ, Kemp AH. Anxiety disorders are associated with reduced heart rate variability: a meta-analysis. Front Psychiatry. 2014;5:80.

63. Clamor A, Lincoln TM, Thayer JF, Koenig J. Resting vagal activity in schizophrenia: meta-analysis of heart rate variability as a potential endophenotype. Br J Psychiatry. 2016;208(1):9-16.

64. Goehler LE, Gaykema RP, Opitz N, Reddaway R, Badr N, Lyte M. Activation in vagal afferents and central autonomic pathways: early responses to intestinal infection with Campylobacter jejuni. Brain Behav Immun. 2005;19(4):334 44. https://doi.org/10.1016/j.bbi.2004.09.002.

65. Bercik P, Park A, Sinclair D, Khoshdel A, Lu J, Huang X, et al. The anxiolytic effect of Bifidobacterium longum NCC3001 involves vagal pathways for gut-brain communication. Neurogastroenterol Motility. 2011;23(12):1132-9.

66. Malick M, Gilbert K, Daniel J, Arseneault-Breard J, Tompkins T, Godbout R, et al. Vagotomy prevents the effect of probiotics on caspase activity in a model of postmyocardial infarction depression. Neurogastroenterol Motility. 2015;27(5):663-71.

67. Bravo JA, Forsythe P, Chew MV, Escaravage E, Savignac HM, Dinan TG, et al. Ingestion of Lactobacillus strain regulates emotional behavior and central GABA receptor expression in a mouse via the vagus nerve. Proc Natl Acad Sci. 2011;108(38):16050-5.

68. Kessler RC, Aguilar-Gaxiola S, Alonso J, Chatterji S, Lee S, Ormel J, et al. The global burden of mental disorders: an update from the WHO World Mental Health (WMH) surveys. Epidemiol Psichiatr Soc. 2009;18(1):23-33. https://doi.org/10.1017/s1121189x00001421.

69. Valles-Colomer M, Falony G, Darzi Y, Tigchelaar EF, Wang J, Tito RY, et al. The neuroactive potential of the human gut microbiota in quality of life and depression. Nat Microbiol. 2019;4(4): 623-32. https://doi.org/10.1038/s41564-018-0337-x.

70. Pirbaglou M, Katz J, de Souza RJ, Stearns JC, Motamed M, Ritvo P. Probiotic supplementation can positively affect anxiety and depressive symptoms: a systematic review of randomized controlled trials. Nutrition Research (New York, NY). 2016;36(9):889-98. https://doi.org/10.1016/j.nutres.2016.06.009.

71. Wallace CJK, Milev R. The effects of probiotics on depressive symptoms in humans: a systematic review. Ann General Psychiatry. 2017;16:14. https://doi.org/10.1186/s12991-017-0138-2.

72. Ng QX, Peters C, Ho CYX, Lim DY, Yeo WS. A meta-analysis of the use of probiotics to alleviate depressive symptoms. J Affect Disord. 2018;228:13-9. https://doi.org/10.1016/j.jad.2017.11.063.

73. Huang R, Wang $\mathrm{K}, \mathrm{Hu}$ J. Effect of probiotics on depression: a systematic review and meta-analysis of randomized controlled trials. Nutrients. 2016;8(8):483. https://doi.org/10.3390/nu8080483.

74. Sanders ME. Impact of probiotics on colonizing microbiota of the gut. J Clin Gastroenterol. 2011;45:S115-S9.

75. Reis DJ, Ilardi SS, Punt SEW. The anxiolytic effect of probiotics: a systematic review and meta-analysis of the clinical and preclinical literature. PLoS One. 2018;13(6):e0199041. https://doi.org/10. 1371/journal.pone.0199041.

76. Foster JA, McVey Neufeld KA. Gut-brain axis: how the microbiome influences anxiety and depression. Trends Neurosci. 2013;36(5):305-12. https://doi.org/10.1016/j.tins.2013.01.005.

77. Hadizadeh M, Hamidi GA, Salami M. Probiotic supplementation improves the cognitive function and the anxiety-like behaviors in the stressed rats. Iran J Basic Med Sci. 2019;22(5):506-14.
78. Dinan TG, Borre YE, Cryan JF. Genomics of schizophrenia: time to consider the gut microbiome? Mol Psychiatry. 2014;19:1252-7. https://doi.org/10.1038/mp.2014.93.

79. Nemani K, Ghomi RH, McCormick B, Fan X. Schizophrenia and the gut-brain axis. Prog Neuro-Psychopharmacol Biol Psychiatry. 2015;56:155-60.

80. Dickerson F, Severance E, Yolken R. The microbiome, immunity, and schizophrenia and bipolar disorder. Brain Behav Immun. 2017;62:46-52.

81. Zheng P, Zeng B, Liu M, Chen J, Pan J, Han Y, et al. The gut microbiome from patients with schizophrenia modulates the glutamate-glutamine-GABA cycle and schizophrenia-relevant behaviors in mice. Science Advances. 2019;5(2):eaau8317. https:// doi.org/10.1126/sciadv.aau8317.

82. Ng QX, Soh AYS, Venkatanarayanan N, Ho CYX, Lim DY, Yeo WS. A systematic review of the effect of probiotic supplementation on schizophrenia symptoms. Neuropsychobiology. 2019;78(1):1-6.

83. Jackson C. Trends in the use of complementary health approaches among adults in the United States: new data. Holist Nurs Pract. 2015;29(3):178-9.

84. Crook N, Ferreiro A, Gasparrini AJ, Pesesky MW, Gibson MK, Wang B, et al. Adaptive strategies of the candidate probiotic $E$. coli Nissle in the mammalian gut. Cell Host \& Microbe. 2019;25(4): 499-512. e8.

85. Kelly JR, Allen AP, Temko A, Hutch W, Kennedy PJ, Farid N, et al. Lost in translation? The potential psychobiotic Lactobacillus rhamnosus (JB-1) fails to modulate stress or cognitive performance in healthy male subjects. Brain Behav Immun. 2017;61:50-9. https://doi.org/10.1016/j.bbi.2016.11.018.

86. Benton D, Williams C, Brown A. Impact of consuming a milk drink containing a probiotic on mood and cognition. Eur J Clin Nutr. 2007;61(3):355-61. https://doi.org/10.1038/sj.ejcn.1602546.

87. Firth J, Stubbs B, Teasdale SB, Ward PB, Veronese N, Shivappa N, et al. Diet as a hot topic in psychiatry: a population-scale study of nutritional intake and inflammatory potential in severe mental illness. World Psychiatry. 2018;17(3):365-7. https://doi.org/10.1002/ wps.20571.

88. Cussotto S, Clarke G, Dinan TG, Cryan JF. Psychotropics and the microbiome: a chamber of secrets. Psychopharmacology. 2019;236(5):1411-32. https://doi.org/10.1007/s00213-019-5185-8 This review highlights differential effects of psychotropic medication on the gut microbiota.

89. Maier L, Pruteanu M, Kuhn M, Zeller G, Telzerow A, Anderson $\mathrm{EE}$, et al. Extensive impact of non-antibiotic drugs on human gut bacteria. Nature. 2018;555(7698):623-8. https://doi.org/10.1038/ nature25979.

90. Davey KJ, Cotter PD, O'Sullivan O, Crispie F, Dinan TG, Cryan JF, et al. Antipsychotics and the gut microbiome: olanzapine-induced metabolic dysfunction is attenuated by antibiotic administration in the rat. Transl Psychiatry. 2013;3:e309. https://doi.org/10.1038/tp. 2013.83.

91. Cussotto S, Strain CR, Fouhy F, Strain RG, Peterson VL, Clarke G, et al. Differential effects of psychotropic drugs on microbiome composition and gastrointestinal function. Psychopharmacology. 2019;236(5):1671-85. https://doi.org/10.1007/s00213-018-50065.

92. Zmora N, Zilberman-Schapira G, Suez J, Mor U, Dori-Bachash M, Bashiardes S, et al. Personalized gut mucosal colonization resistance to empiric probiotics is associated with unique host and microbiome features. Cell. 2018;174(6):1388-405.e21.

93. Jacka FN. Nutritional psychiatry: where to next? EBioMedicine. 2017;17:24-9. https://doi.org/10.1016/j.ebiom.2017.02.020.

Publisher's Note Springer Nature remains neutral with regard to jurisdictional claims in published maps and institutional affiliations. 